\title{
Les méthodes chromatographiques et électrophorétiques récentes d'analyse des phosphates inorganiques
}

\author{
R. Rosset*, F. Hui, J. Xie, H. Kolodziejczyk et B. Bayri \\ Laboratoire de Chimie analytique des Processus industriels, École Supérieure de Physique et Chimie \\ Industrielles de la Ville de Paris, 10, rue Vauquelin, 75005 Paris, France
}

\begin{abstract}
Following a classification of phosphates according to modern rules of nomenclature, the separation of complex mixtures of linear and cyclic condensed phosphates is described. Three methods are evaluated: ion exchange liquid chromatography with chloride concentration gradient, ion chromatography associated with conductometric detection which allows rapid separations $(\approx 30$ minutes), capillary electrophoresis with indirect absorptiometric detection, this technique being, for the moment, restricted to the first terms of phosphates (ortho, di and triphosphate).
\end{abstract}

Key words. Linear and cyclic condensed phosphates - ion exchange liquid chromatography - ion chromatography - conductometric detection - capillary electrophoresis.

\section{Introduction}

Les oxoacides du phosphore au degré d'oxydation $+\mathrm{V}$, acide phosphorique, acides phosphoriques condensés (acides polyphosphoriques et polymétaphosphoriques) et leurs sels constituent des produits industriels d'une très grande importance dans des domaines aussi variés que la détergence, les engrais, l'inhibition de la corrosion et de l'entartrage, les adjuvants alimentaires, etc.

Ces produits sont des mélanges complexes d'espèces qui peuvent être très nombreuses en raison des multiples possibilités structurales qu'offre la condensation, par déshydratation progressive, de l'acide phosphorique $\mathrm{H}_{3} \mathrm{PO}_{4}$ au pentoxyde $\mathrm{P}_{2} \mathrm{O}_{5}$ (qui existe, en fait, à l'état de dimère, $\mathrm{P}_{4} \mathrm{O}_{10}$ ).

$\mathrm{Au}$ fil des années, de multiples méthodes chromatographiques en phase liquide ont été imaginées pour caractériser ces produits. Une recherche bibliographique informatisée* ne révèle pas moins de 291 références mais dont la plupart n'ont plus, aujourd'hui, qu'un intérêt historique. Nous avons limité la présente publication aux résultats les plus récents en y incluant l'électrophorèse capillaire qui n'a pas encore atteint, pour l'analyse des phosphates, le degré de perfection de la chromatographie en phase liquide mais dont le développement actuel est prometteur.

\section{Classification des phosphates}

Les oxoacides du phosphore au degré d'oxydation $+\mathrm{V}$ sont classés en trois groupes [1-3] :

- les phosphates linéaires,

- les phosphates cycliques,

- les ultraphosphates.

Les ultraphosphates, dont nous donnons quelques exemples, ne sont stables qu'à l'état solide; en solution aqueuse, ils se décomposent en phosphates linéaires et (ou) cycliques et, par suite, ils sont, de fait, exclus de la présente étude. En regard de chaque composé nous avons indiqué l'abréviation correspondante.

\section{Phosphates linéaires}

Les premiers termes sont (forme acide) :

- l'acide monophosphorique, encore appelé acide phosphorique ou ortho-phosphorique :

$$
\begin{array}{ll}
\mathrm{H}_{3} \mathrm{PO}_{4} & \mathrm{HO} \searrow \\
& \mathrm{HO}
\end{array}
$$

- l'acide diphosphorique (pyrophosphorique) :<smiles>[20PH2]</smiles><smiles>O=P(O)(O)OP(=O)(O)O</smiles>

$\mathrm{P}_{2}$

- l'acide triphosphorique (le sel de sodium correspondant, utilisé en détergence, est appelé tripolyphosphate de sodium) :<smiles>O=P(O)(O)OP(=O)(O)OP(=O)(O)O</smiles>

puis, on a les polyphosphates de formule générale :

$$
\mathrm{H}_{n+2} \mathrm{P}_{n} \mathrm{O}_{3 n+1} \quad \mathrm{P}_{n}
$$

dont on a identifié les espèces par chromatographie en phase liquide au moins jusqu'à $n=36$. L'acide tétraphosphorique $\mathrm{H}_{6} \mathrm{P}_{4} \mathrm{O}_{13}(n=4)$ et ses sels sont des produits commerciaux. Lorsque $n \leq 5$ on parle d'oligophosphates.

\footnotetext{
* Correspondance et tirés-à-part. Le résultat de cette recherche sous la forme de la liste des publications avec leur titre, etc. est disponible sur demande auprès des auteurs. Reçu le 23 septembre 1997 ; révisé le 11 décembre 1997; accepté le 23 décembre 1997.
} 
Le tableau I résume la nomenclature des phosphates linéaires.

Tableau I. Nomenclature des phosphates linéaires

\begin{tabular}{lcc}
\hline & \multicolumn{2}{c}{ Polyphosphates } \\
\hline$n$ & Anions : $\left[\mathrm{P}_{n} \mathrm{O}_{3 n+1}\right]^{(n+2)-}$ & Nom du sel \\
1 & $\mathrm{PO}_{4}^{3-}$ & monophosphates \\
2 & {$\left[\mathrm{P}_{2} \mathrm{O}_{7}\right]^{4-}$} & diphosphates \\
3 & {$\left[\mathrm{P}_{3} \mathrm{O}_{10}\right]^{5-}$} & triphosphates \\
4 & {$\left[\mathrm{P}_{4} \mathrm{O}_{13}\right]^{6-}$} & tétraphosphates \\
5 & {$\left[\mathrm{P}_{5} \mathrm{O}_{16}\right]^{7-}$} & pentaphosphates \\
$n>5$ & {$\left[\mathrm{PO}_{3}\right]_{n}^{(n+2)-*}$} & polyphosphates à longue chaîne
\end{tabular}

* ou $\mathrm{P}_{n} \mathrm{O}_{3 n+1}, 1$ devenant cependant négligeable devant $3 n$ lorsque $n$ est grand.

\section{Les phosphates cycliques}

Ils ont, pour formule générale (forme acide) :

$$
\mathrm{H}_{n} \mathrm{P}_{n} \mathrm{O}_{3 n} \quad \text { ou } \quad\left(\mathrm{HPO}_{3}\right)_{n} \text {. }
$$

Pendant longtemps on les nommait en ajoutant, pour les distinguer des précédents, le préfixe meta. Une terminologie plus claire avec le préfixe cyclo est, aujourd'hui, utilisée.

Les deux premiers termes ont été précisément identifiés :

- l'acide cyclotriphosphorique (autrefois trimétaphosphorique) :

$$
\mathrm{H}_{3} \mathrm{P}_{3} \mathrm{O}_{9} \quad \text { ou } \quad\left(\mathrm{HPO}_{3}\right)_{3} \quad \mathrm{P}_{3 m}
$$<smiles>O=P1(O)OP(=O)(O)OP(=O)(O)O1</smiles>

- l'acide cyclotétraphosphorique (autrefois tétramétaphosphorique) :

$$
\mathrm{H}_{4} \mathrm{P}_{4} \mathrm{O}_{12} \quad \text { ou } \quad\left(\mathrm{HPO}_{3}\right)_{4} \quad \mathrm{P}_{4 m}
$$<smiles>O=P1(O)OP(=O)(O)OP(=O)(O)OP(=O)(O)O1</smiles>

On a ensuite les cyclopolyphosphates de formule générale (pour la forme acide) :

$$
\left(\mathrm{HPO}_{3}\right)_{n} \quad \mathrm{H}_{n} \mathrm{P}_{n} \mathrm{O}_{3 n} \quad \mathrm{P}_{n m}
$$

Le Tableau II donne la nomenclature actuelle des phosphates cycliques :
Tableau II. Nomenclature des cyclophosphates.

\begin{tabular}{lcc}
\hline & \multicolumn{2}{c}{ Cyclophosphates } \\
$n$ & Anions : $\left[\mathrm{P}_{n} \mathrm{O}_{3 n}\right]^{n-}$ & Nom du sel \\
3 & {$\left[\mathrm{P}_{3} \mathrm{O}_{9}\right]^{3-}$} & cyclotriphosphate \\
4 & {$\left[\mathrm{P}_{4} \mathrm{O}_{12}\right]^{4-}$} & cyclotétraphosphate \\
5 & {$\left[\mathrm{P}_{5} \mathrm{O}_{15}\right]^{5-}$} & cyclopentaphosphate \\
6 & {$\left[\mathrm{P}_{6} \mathrm{O}_{18}\right]^{6-}$} & cyclohexaphosphate \\
8 & {$\left[\mathrm{P}_{8} \mathrm{O}_{24}\right]^{8-}$} & cyclooctaphosphate \\
9 & {$\left[\mathrm{P}_{9} \mathrm{O}_{27}\right]^{--}$} & cyclononaphosphate \\
10 & {$\left[\mathrm{P}_{10} \mathrm{O}_{30}\right]^{10-}$} & cyclodécaphosphate \\
12 & {$\left[\mathrm{P}_{12} \mathrm{O}_{36}\right]^{12-}$} & cyclododécaphosphate \\
\hline
\end{tabular}

Le produit commercialisé sous le nom d'hexamétaphosphate ne correspond pas à $n=6$ mais à un mélange complexe d'acides cycliques condensés avec un $n$ moyen de 20 .

\section{Les ultraphosphates}

Bien qu'ils ne soient pas stables en solutions aqueuses nous donnons la formule générale de ces anions :

$$
n\left[\mathrm{PO}_{3}\right]^{-}+m \mathrm{P}_{2} \mathrm{O}_{5} \quad \text { ou } \quad\left[\mathrm{P}_{(2 m+n)} \mathrm{O}_{(5 m+3 n)}\right]^{n-} .
$$

Les seuls anions caractérisés sans ambiguïté à l'état solide sont ceux correspondants à $m=1$ dont la formule générale est :

$$
\left[\mathrm{P}_{(n+2)} \mathrm{O}_{(3 n+5)}\right]^{n-} \text {. }
$$

Les anions avec $n=2,3,4,5$ et 6 ont été clairement identifiés, par exemple avec $n=2$ (diultraphosphate), sous la forme acide :<smiles>O=P1(O)OP2(=O)OP(=O)(O)OP(=O)(O1)O2</smiles>

Ces composés sont également appelés phosphates ternaires ou phosphates branchés.

\section{Chromatographie d'échange d'ions}

Nous traiterons de la chromatographie d'échange d'ions proprement dite [4-24] puis, dans une partie distincte de la chromatographie ionique [25-27] qui ne diffère de la précédente que par la mise en œuvre d'une détection conductométrique au lieu de la détection absorptiométrique avec dérivation post-colonne utilisée classiquement.

Dès les premières publications sur le sujet, les auteurs ont choisi une colonne d'un polymère échangeur d'anions de type base forte (à groupements triméthylammonium $\left.-\mathrm{N}\left(\mathrm{CH}_{3}\right)_{3}^{+}\right)$, la séparation des différents phosphates étant obtenue par élution graduée avec une phase mobile constituée d'une solution de chlorure de potassium de concentration croissante [4,5]. Par la suite, les auteurs ont préféré 
utiliser des solutions de chlorure de sodium. La détection est effectuée par absorptiométrie après hydrolyse des phosphates condensés en monophosphate $\mathrm{PO}_{4}^{3-}$ et formation d'un hétéropolyanion, le complexe phosphovanadomolybdate de couleur jaune. La méthode a été automatisée dès 1961 par Lundgren et al. [6] puis par Benz [7,8].

En 1976, Fukuda et al. [9] ont observé que l'anion diphosphate présentait une traîne qui a été attribuée à l'hydrolyse du diphosphate en monophosphate dans la colonne chromatographique. Cette réaction d'hydrolyse est catalysée par les cations de métaux lourds présents, en tant qu'impuretés, dans l'éluant (solution de chlorure de sodium). On peut empêcher cette hydrolyse en ajoutant à la phase mobile un complexant de ces cations, l'anion de l'acide éthylènediaminetétracétique (EDTA).

Une excellente séparation (pour l'époque, où l'effluent est fractionné au moyen d'un collecteur de fractions et chaque fraction analysée par absorptiométrie) des phosphates linéaires de $\mathrm{PO}_{4}^{3-}$ à $\left[\mathrm{P}_{8} \mathrm{O}_{25}\right]^{10-}$ est représentée figure 1 .

La volonté d'automatiser la séparation et de permettre la détection en continu des espèces éluées a conduit à développer deux types de détecteurs avec dérivation postcolonne: les systèmes à air segmenté (AutoAnalyzer II de TECHNICON) comme dans [12] et les systèmes à flux continu comme dans $[13,17]$. La dérivation post-colonne est toujours fondée sur la formation d'un hétéropolyanion par réaction de l'anion orthophosphate avec le molybdène (VI). Le plus souvent le complexe phosphomolybdate de couleur jaune est réduit par l'acide ascorbique pour donner un composé de couleur bleue dont on mesure l'absorbance. Les phosphates condensés sont hydrolysés en orthophosphate en milieu acide fort. Avec l'AutoAnalyzer II la coloration apparaît 18 minutes après la sortie de l'espèce à détecter de la colonne chromatographique.

Avec le système à flux continu (FIA : flow injection analysis) on réduit le diamètre du serpentin de réaction et on augmente le débit ce qui crée un écoulement turbulent. La figure 2 représente le système développé par Ohashi et al. [13]. L'hydrolyse est réalisée de manière accélérée à $140{ }^{\circ} \mathrm{C}$.

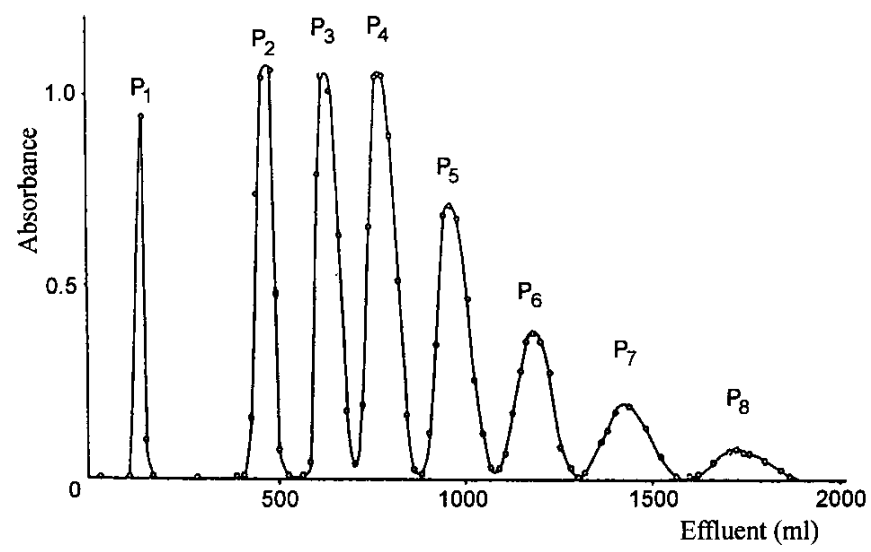

Fig. 1. Séparation par chromatographie d'échange d'ions des anions phosphates $\mathrm{P}_{1}$ à $\mathrm{P}_{8}$. La phase mobile est un mélange de chlorure de sodium et d'EDTA. Détection absorptiométrique par formation du phosphovanadomolybdate d'ammonium. D'après [10].
L'optimisation de la séparation des phosphates a fait l'objet de très nombreux travaux [10-23] dont on peut résumer les conclusions comme suit :

- l'emploi d'un polymère échangeur d'anions à faible taux de pontage ( $4 \%$ de divinylbenzène) permet de séparer les phosphates linéaires condensés jusqu'à $n=36$ (les cyclophosphates s'insérant dans l'élution des phosphates linéaires) ; l'emploi d'un polymère échangeur d'anions à taux de pontage plus élevé ( $8 \%$ de divinylbenzène) donne une bonne séparation des phosphates linéaires jusqu'à $n=11$;

- l'addition d'EDTA à la phase mobile (solutions de chlorure de sodium) est indispensable pour éviter l'hydrolyse des phosphates condensés, tout particulièrement de l'anion diphosphate, au cours de l'élution chromatographique ;

- en diminuant la concentration du chlorure de sodium dans la phase mobile la résolution de la séparation est améliorée mais le temps d'analyse augmente;

- une augmentation de la température de la colonne (30 à $40^{\circ} \mathrm{C}$ en général) améliore la résolution.

Nous avons représenté figure 3 la séparation de phosphates linéaires jusqu'à $n=30$ avec un gradient de concentration en chlorure de sodium convexe de 0,22 à $0,53 \mathrm{~mol}$ $\mathrm{L}^{-1}$. La durée totale de la séparation est de 6 heures mais la résolution est remarquable. Figure 4 on a représenté la séparation de mélanges plus simples mais contenant des cyclophosphates. Figure 5 on a une séparation de phosphates linéaires (jusqu'à $n=20$ environ) et de cyclophosphates $(3 \mathrm{~m}, 4 \mathrm{~m}, 6 \mathrm{~m}$ sur la figure, en utilisant l'ancienne nomenclature des métaphosphates ce qui correspond à $n=3,4$ et 6 dans le tableau II).

En utilisant des polymères échangeurs d'ions de fine granulométrie et une détection en flux continu la durée de la séparation est réduite à 45 minutes (Fig. 6 où des données détaillées sont fournies sur les conditions expérimentales, la publication correspondante [24] étant en japonais).

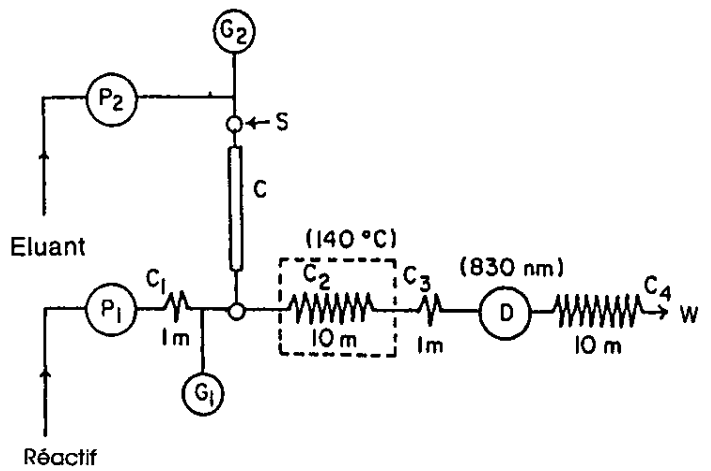

Fig. 2. Couplage d'un chromatographe en phase liquide avec un système à flux continu sous haute pression comme détecteur. $\mathrm{P}_{1}$ et $\mathrm{P}_{2}$ : pompes; $\mathrm{G}_{1}$ et $\mathrm{G}_{2}$ : capteurs de mesure de pression; $\mathrm{C}_{1}$ : serpentin pré-réaction; $\mathrm{C}_{2}$ : serpentin de réaction; $\mathrm{C}_{3}$ : serpentin de refroidissement $; \mathrm{C}_{4}$ : serpentin de contre-pression (évite la formation de bulles d'air dans le serpentin de réaction en dépit de la température élevée qui y règne : $140{ }^{\circ} \mathrm{C}$ ); $\mathrm{D}:$ détecteur ; $\mathrm{C}:$ colonne chromatographique; $\mathrm{S}$ : injection de l'échantillon; W : à l'évier (waste). Les longueurs des serpentins sont indiquées en mètres. La détection se fait à $830 \mathrm{~nm}$. D'après [13]. 


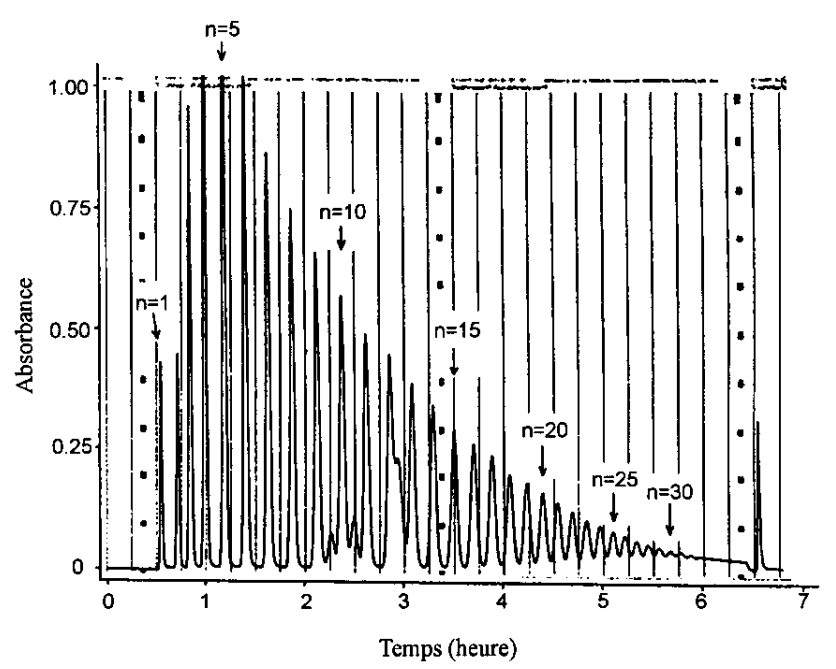

Fig. 3. Séparation de phosphates linéaires de $n=1$ à $n=30$ environ par élution graduée avec un gradient convexe de chlorure de sodium de 0,22 à $0,53 \mathrm{~mol} \mathrm{~L}^{-1}$, EDTA $5 \times 10^{-3} \mathrm{~mol} \mathrm{~L}^{-1}, \mathrm{pH} 10$. Polymère échangeur d'anions (HITACHI 2630) à $4 \%$ de divinylbenzène. Longueur de la colonne : $10 \mathrm{~cm}$; diamètre intérieur : $9 \mathrm{~mm}$. Température : $31 \pm 1{ }^{\circ} \mathrm{C}$. Débit de la phase mobile : $1 \mathrm{~mL}$ $\min ^{-1}$. D'après [12].

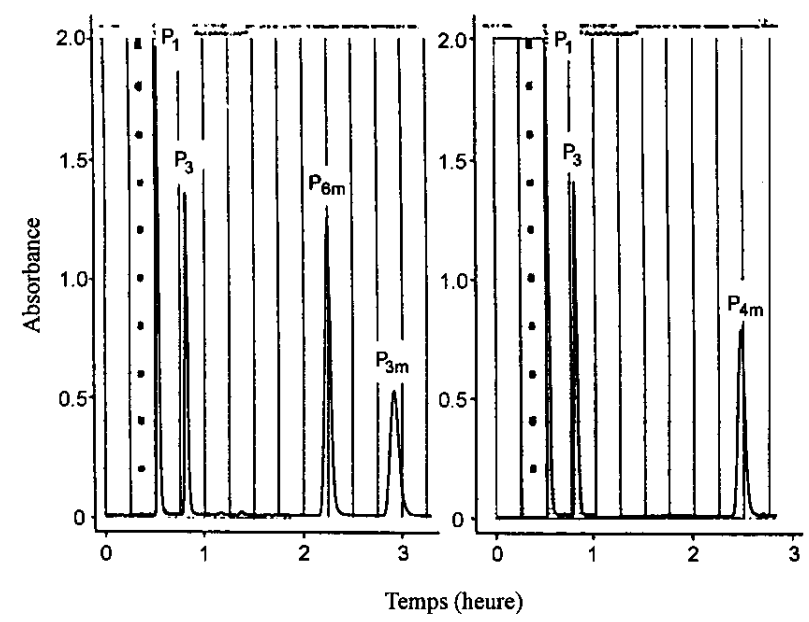

Fig. 4. Séparation de phosphates linéaires $(n=1,3)$ et cycliques $(n=3$ et 6 ou 4$)$. Conditions identiques à celles de la figure 3. D'après [12].

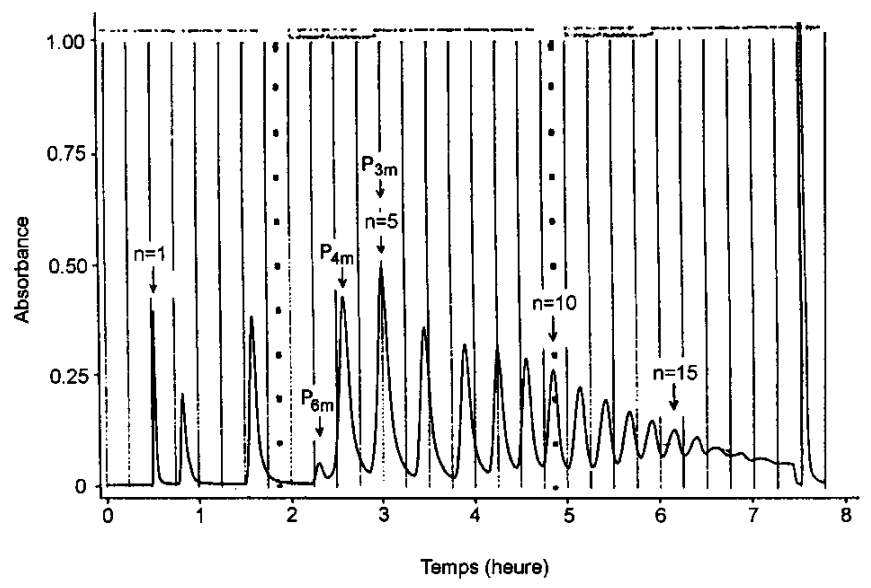

Fig. 5. Séparation de phosphates linéaires ( $n=1$ à 20 environ) et cycliques $(n=3,4,6)$. Conditions identiques à celles de la figure 3 sauf $\mathrm{pH}$ de la phase mobile : 4,4. D'après [12].
Se fondant sur une approche théorique de Jandera et Churácek [28], Baba et al. [19-21] ont proposé une expression théorique du temps de rétention des phosphates condensés :

$$
\left.t_{\mathrm{R}}=t_{0}+\frac{1}{u}\left\{\left(\frac{1}{B^{\prime}}\right)\left[(x b+) B^{\prime} \mathrm{at}_{0} u+C_{\mathrm{i}}^{(x b+)}\right) /\right]^{\frac{1}{x b+1}}-\frac{C_{\mathrm{i}}^{1 / \lambda}}{B^{\prime}}\right\}
$$

$t_{0}$ : temps de rétention nulle

$u$ : vitesse linéaire de la phase mobile

$a, b$ : constantes pour un soluté donné obtenues par le tracé de $\log k^{\prime}=f(\log C)$ dans le cas d'une élution isocratique (on a alors $k^{\prime}=a C^{-b}, k^{\prime}$ étant le facteur de rétention, $C$ la concentration de la phase mobile en l'espèce éluante)

$C_{\mathrm{i}}$ : concentration initiale de l'éluant lors de l'élution graduée $(t=0)$.

$B^{\prime}=B / u, B$ étant, comme $x$, un paramètre ajustable qui caractérise le profil du gradient. On a :

$$
\begin{gathered}
C=\left(C_{\mathrm{i}}^{1 / x}+B t\right)^{x} \\
B=\left(C_{\mathrm{f}}^{1 / x}+C_{\mathrm{i}}^{1 / x}\right) / t_{\mathrm{f}}
\end{gathered}
$$

$C$ : concentration au temps $t$

$C_{\mathrm{f}}$ : concentration finale de l'éluant $\left(t=t_{\mathrm{f}}\right)$

$x$ : caractérise le profil du gradient : $x=1$ pour un profil linéaire ; $x<1$ pour un profil convexe ; $x>1$ pour un profil concave.

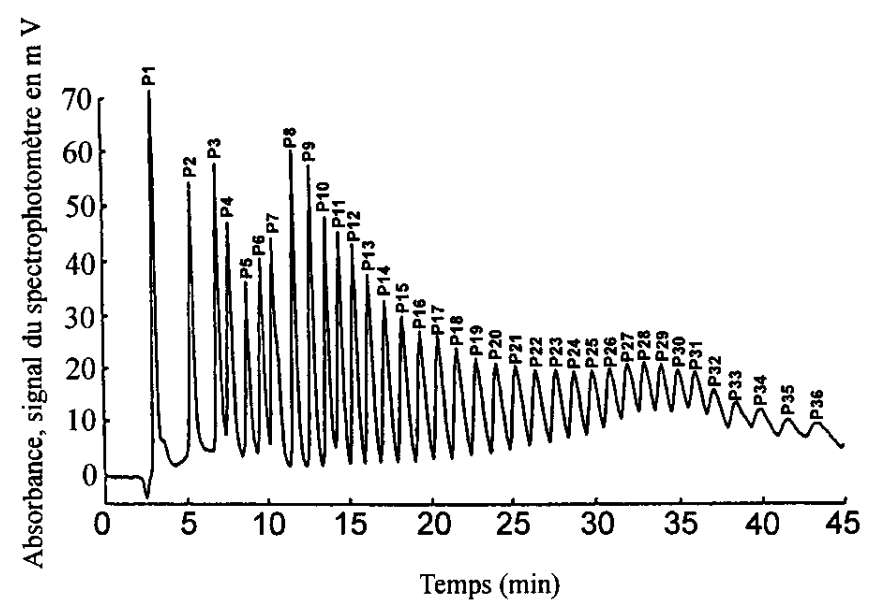

Fig. 6. Séparation de phosphates linéaires (de $n=1$ à $n=36$ ). Phases stationnaires : polymère échangeur d'anions Excelpak ICSA23 et ICS-A2G. Colonne: longueur $75 \mathrm{~mm}$, diamètre intérieur $4,6 \mathrm{~mm}$ pour le premier échangeur, $25 \mathrm{~mm} \times 2,7 \mathrm{~mm}$ pour le second. Phase mobile : A : EDTA, sel tétrasodique $1,5 \times 10^{-3} \mathrm{~mol}$ $\mathrm{L}^{-1}$; $\mathrm{B}$ : EDTA, sel tétrasodique $1,5 \times 10^{-3} \mathrm{~mol} \mathrm{~L}^{-1}$, chlorure de sodium $0,2 \mathrm{~mol} \mathrm{~L}^{-1}$. Gradient : $\mathrm{A}$ : 80 à $40 \%$ de 0 à 10 minutes ; $\mathrm{A}: 40$ à $20 \%$ de 10 à 30 minutes; $\mathrm{A}: 20 \%$ de 30 à 45 minutes. Température de la colonne : $40^{\circ} \mathrm{C}$. D'après [24]. 
Baba et al. [19-21, 23] ont, au moyen d'un progiciel, calculé les temps de rétention de phosphates condensés linéaires et cycliques pour des conditions chromatographiques $\left(t_{0}, u\right)$ et des gradients donnés et ont comparé les chromatogrammes théoriques obtenus à des chromatogrammes expérimentaux. L'accord est excellent aussi bien pour les phosphates linéaires que pour les phosphates cycliques comme le montrent les figures 7 et 8 .

La chromatographie en phase liquide des phosphates est donc parvenue à un haut degré de perfection. Deux améliorations ont été proposées : le remplacement des solutions de chlorure de sodium utilisées comme phase mobile par un mélange de nitrate de calcium et de sulfate de magnésium: la force éluante est renforcée par la formation de complexes entre les anions phosphates et les cations $\mathrm{Ca}^{2+}$ et $\mathrm{Mg}^{2+}$ et la phase mobile est moins corrosive pour l'appareillage [29].

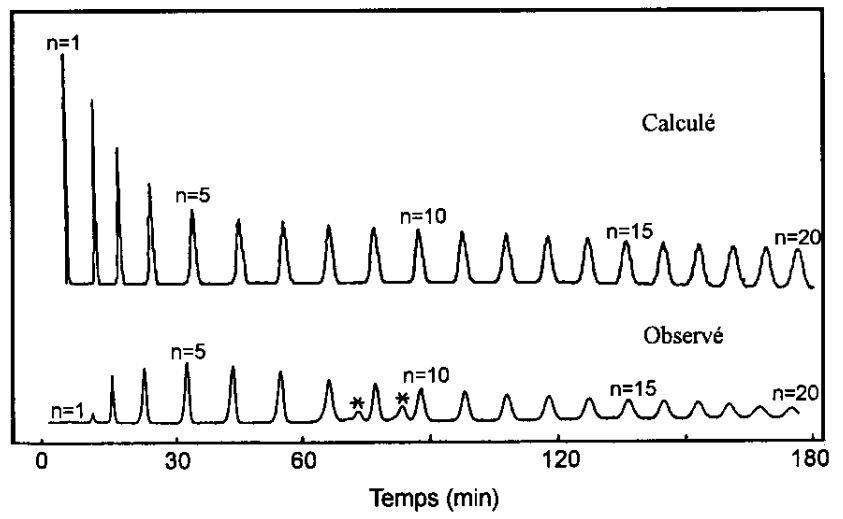

Fig. 7. Chromatogrammes calculés et observés pour les phosphates condensés linéaires de $n=1$ à $n=20(\bar{n}=10)$. Sur le chromatogramme expérimental on observe deux pics supplémentaires entre $n=8$ et $n=10$ (signalés par un astérisque) qui correspondent à la présence, dans l'échantillon, de phosphates cycliques. D'après [20].
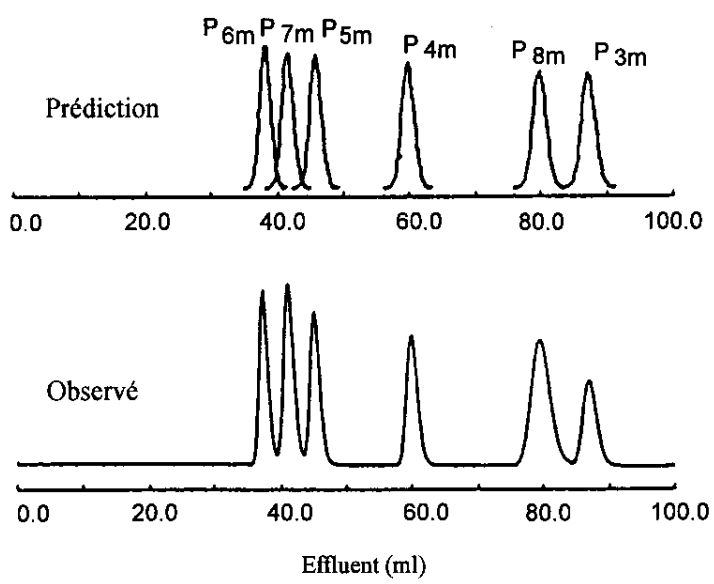

Fig. 8. Chromatogrammes calculés et observés pour six phosphates cycliques avec un gradient linéaire de concentration $(x=1)$ de $C_{\mathrm{i}}=0,4$ à $C_{\mathrm{f}}=0,5 \mathrm{~mol} \mathrm{~L}^{-1}$ en 350 minutes $\left(t_{\mathrm{f}}\right)$.
En ce qui concerne la détection on a proposé de remplacer l'absorptiométrie par une détection par spectrométrie d'absorption atomique : l'élution des phosphates est effectuée par une solution de chlorure de magnésium de concentration connue; il se forme des complexes entre le cation $\mathrm{Mg}^{2+}$ et les anions phosphate; la concentration du magnésium dans l'effluent est liée à celle des anions phosphate que l'on peut ainsi déterminer [30]. Mais, l'appareillage nécessaire est moins courant que le spectrophotomètre que l'on utilise avec la méthode au bleu de molybdène.

\section{Chromatographie ionique}

La chromatographie ionique associe une séparation par chromatographie d'échange d'ions à une méthode de détection appropriée, conductométrique ou absorptiométrique (par diminution d'absorbance) [31]. Deux méthodes ont été proposées.

Danielson et al. [26] séparent un mélange assez simple de trois phosphates condensés linéaires $\left(\mathrm{P}_{2}, \mathrm{P}_{3}, \mathrm{P}_{4}\right)$ et d'un phosphate cyclique $\left(\mathrm{P}_{3 m}\right)$ sur une colonne d'un polymère échangeur d'anions de type base forte de faible capacité d'échange $\left(0,2 \mathrm{meq} \mathrm{g}^{-1}\right)$ Hamilton PRP-X 100 en utilisant comme éluant une solution de naphtalène 1, 3, 6-trisulfonate de sodium $10^{-2} \mathrm{~mol} \mathrm{l}^{-1}$ dans le mélange eau-acétonitrile $95: 5(\mathrm{v} / \mathrm{v})$. Le faible pourcentage d'acétonitrile limite l'interaction hydrophobe entre le noyau naphtalène et les noyaux aromatiques du copolymère styrène-divinylbenzène.

L'anion naphtalènetrisulfonate porte trois charges négatives et a une force éluante élevée permettant l'élution des anions phosphate. Par ailleurs, il absorbe fortement dans l'ultraviolet tandis que les anions phosphate n'absorbent pas; on peut donc effectuer la détection par diminution d'absorbance. Les limites de détection obtenues sont données tableau III.

Tableau III. Limites de détection pour des anions phosphate condensés séparés par chromatographie ionique avec détection par diminution d'absorbance. D'après [26].

\begin{tabular}{lcc}
\hline Espèces chromatographiées & \multicolumn{2}{c}{ Limite de détection } \\
& $m g$ L $^{-1^{*}}$ & $n g$ \\
\hline diphosphate & 1 & 20 \\
cyclotriphosphate & 0,1 & 2 \\
triphosphate & 1 & 20 \\
tétraphosphate & 2,5 & 50 \\
\hline
\end{tabular}

* volume injecté: $20 \mu \mathrm{L}$.

Remarques. a) le mélange de phosphates condensés sépa rés par Danielson n'est pas très complexe mais les auteurs les étudient en mélange avec des anions polycarboxyliques (nitrilotriacétate, éthylènediaminetétracétate, citrate) tels qu'on peut les rencontrer dans des détergents ou des produits alimentaires.

b) l'anion naphtalènetrisulfonate a une taille importante et, de ce fait, une mobilité faible en solution; par suite, on peut 
également détecter par conductométrie sans suppresseur de la conductivité de l'éluant. Toutefois, la ligne de base est perturbée et la méthode est d'une application plus délicate que l'absorptiométrie par diminution d'absorbance.

Une séparation beaucoup plus performante a été publiée par Stover et al. [27] en utilisant comme phase stationnaire un échangeur d'ions (Dionex PAX-100) constitué de particules de latex de $60 \mathrm{~nm}$ de diamètre fonctionnalisées par des groupements ammonium quaternaire greffées sur des particules de $8,5 \mu \mathrm{m}$ de diamètre d'un copolymère éthylvinybenzène-divinylbenzène [30]. La phase mobile est une solution d'hydroxyde de sodium dans le mélange eau-méthanol $95: 5(\mathrm{v} / \mathrm{v})$.

On opère par élution graduée de $\mathrm{NaOH} 4 \times 10^{-2}$ à $0,3 \mathrm{~mol}$ $\mathrm{L}^{-1}$ de 0 à 20 minutes puis à concentration finale constante pendant 10 minutes. La détection est effectuée après suppression de la conductivité de la phase mobile [31], par conductométrie. La séparation obtenue est remarquable comme en témoigne la figure 9 où l'on détecte les phosphates linéaires condensés jusqu'à $n \approx 30$ environ, la durée de la séparation n'étant que d'une demi-heure ; la limite de détection est de $1 \mathrm{ng}$ injecté de l'échantillon à étudier.

On peut évidemment considérer que les séparations tétraphosphate-cyclotriphosphate et pentaphosphate-cyclotétraphosphate sont insuffisantes. Chacune de ces séparations peut être améliorée en utilisant un gradient convexe mais au détriment de la résolution pour l'autre partie du chromatogramme.

Cette méthode a été utilisée pour étudier l'hydrolyse du produit industriel hexamétaphosphate en fonction de la température (Fig. 10). Elle permet également de caractériser les verres de phosphate dont les degrés de polymérisation moyens peuvent être très élevés.

Une difficulté doit cependant être soulignée : l'incertitude où l'on est des coefficients de réponse du conductomètre pour les différents phosphates condensés (alors que la détection classique par hydrolyse des phosphates condensés en orthophosphate et formation de l'anion phosphomolybdate, en étant indépendante de la nature du polyphosphate, convient pour des mesures quantitatives). La solution de ce problème passerait par la préparation d'étalons de phosphates condensés.

Remarque. La difficulté précédente explique la concurrence qui existe entre la chromatographie ionique et la résonance magnétique nucléaire à transformée de Fourier du phos phore 31 laquelle permet de calculer le degré de polyméri sation moyen des phosphates condensés sans qu'il soit nécessaire de disposer d'étalons. Toutefois, la chromatographie apporte des informations très riches sur la distribution des différentes espèces linéaires et cycliques.

\section{Électrophorèse capillaire}

Cette méthode permet généralement des analyses d'une grande rapidité avec une résolution élevée et une faible consommation de phase mobile ; le fait d'utiliser un capillaire est considéré comme plus simple qu'une colonne chromatographique. En mettant en œuvre un électrolyte absorbant dans l'ultraviolet on peut détecter, par diminution

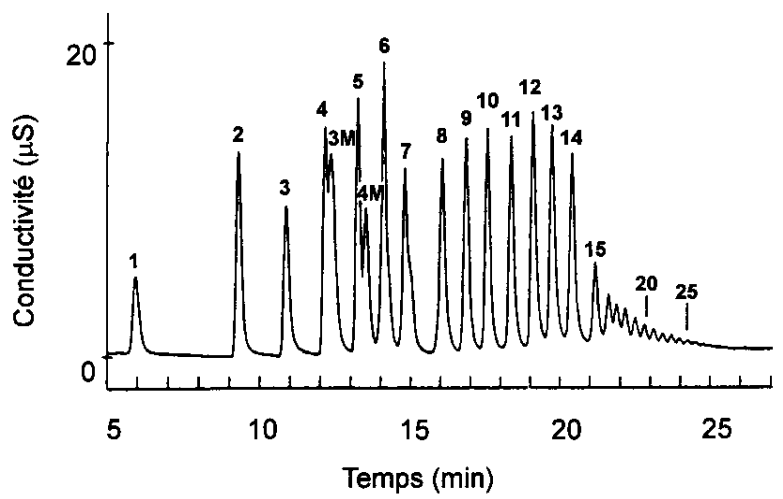

Fig. 9. Séparation par chromatographie ionique avec détection conductométrique des phosphates condensés linéaires (de $n=1$ à $n \approx 30$ ) et cycliques ( $n=3, n=4)$ d'un échantillon d'hexamétaphosphate de sodium. Colonne : longueur : $250 \mathrm{~mm}$; diamètre intérieur : $2 \mathrm{~mm}$; phase stationnaire (Cf. texte). Gradient linéaire (Cf. texte). Concentration de la solution injectée : $0,2 \mathrm{mg} / \mathrm{mL}$. Volume injecté : $25 \mu \mathrm{L}$ (5 ng injecté). Débit : $0,25 \mathrm{~mL} / \mathrm{min}$; température ambiante.
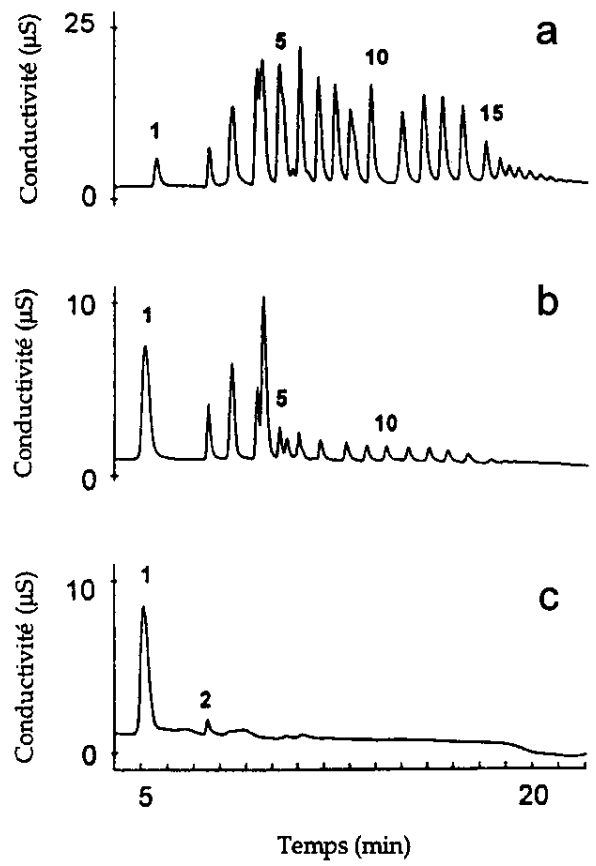

Fig. 10. Etude de l'hydrolyse d'une solution aqueuse à $40 \%$ d'hexamétaphosphate conservée pendant 7 jours à trois températures (a) : température ambiante ; (b) : $49{ }^{\circ} \mathrm{C}$; (c) : $60^{\circ} \mathrm{C}$. A $60{ }^{\circ} \mathrm{C}$ $1^{\prime}$ hydrolyse en orthophosphate (avec une faible quantité de diphosphate) est pratiquement totale. Conditions des séparations identiques à celles de la figure 9 .

d'absorbance, des solutés qui n'absorbent pas dans l'UV ce qui est le cas des phosphates.

Sur ces principes une méthode de séparation des premiers termes des phosphates (orthophosphate, diphosphate, triphosphate) a été publiée par Stover et al. [32]. L'électrolyte est une solution de monohydrogénophtalate de potassium (KHP) $5 \times 10^{-3} \mathrm{~mol} \mathrm{~L}^{-1}, \mathrm{pH} \mathrm{4,2}$. Pour contrebalancer le flux électroosmotique, qui est en sens inverse du déplacement 
des anions sous l'influence du champ électrique appliqué, on ajoute à l'électrolyte un cation ammonium quaternaire, le dodécyltriméthylammonium $\left(\mathrm{C}_{12} \mathrm{H}_{25}\right)\left(\mathrm{CH}_{3}\right)_{3} \mathrm{~N}^{+}$(sous forme bromure) à la concentration $5 \times 10^{-4} \mathrm{~mol} \mathrm{~L}^{-1}$. Il y a alors inversion du flux électroosmotique.

Les mobilités électrophorétiques des espèces considérées ainsi que celle du phtalate varient en fonction du $\mathrm{pH}$ comme indiqué figure 11 . On voit que les différences de mobilité des phosphates ne varient pratiquement pas avec le $\mathrm{pH}$; les trois courbes référencées $\mathrm{O}, \mathrm{P}$ et $\mathrm{T}$ sont sensiblement parallèles. Par suite, la sélectivité de la séparation varie peu avec le $\mathrm{pH}$. En revanche, la mobilité électrophorétique du phtalate varie avec le $\mathrm{pH}$. Celui choisi pour effectuer la séparation est $4,2, \mathrm{pH}$ où le phtalate présente une mobilité électrophorétique intermédiaire par rapport à celles de l'ortho et du diphosphate. C'est à ce $\mathrm{pH}$ que la dissymétrie des pics est minimale. Le triphosphate dont la mobilité est la plus éloignée de celle du phtalate présente la plus grande dissy-

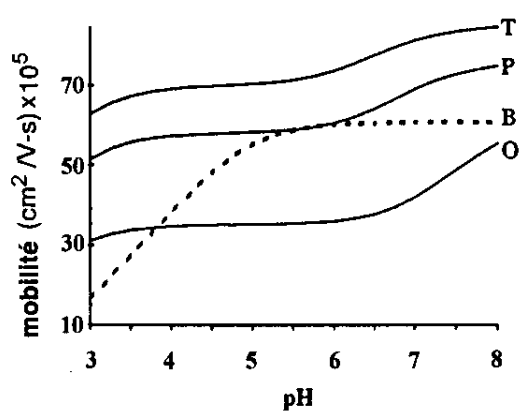

Fig. 11. Variation de la mobilité électrophorétique en fonction du pH. O : orthophosphate ; P : diphosphate (pyrophosphate) ; T : triphosphate; $\mathrm{B}$ : phtalate. D'après [32]

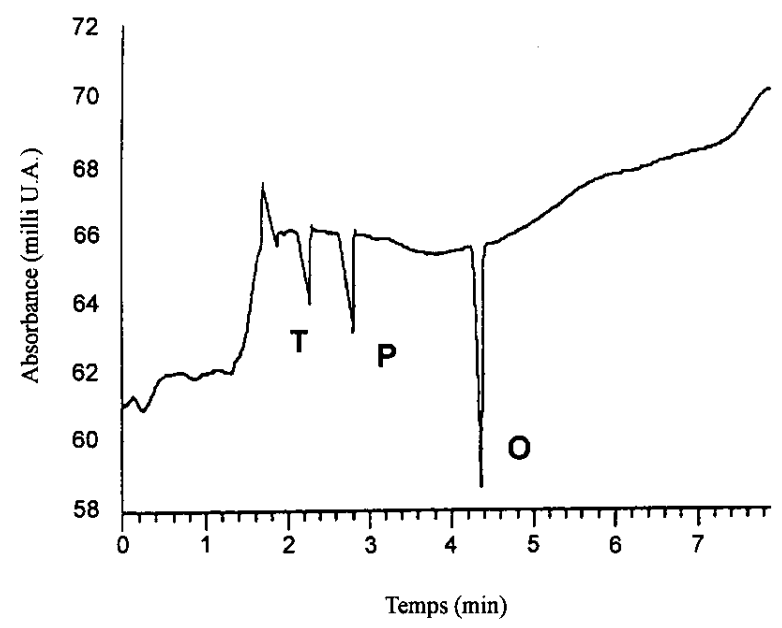

Fig. 12. Séparation des trois phosphates considérés par électrophorèse capillaire. Electrolyte: monohydrogénophtalate de potassium $5 \times 10^{-3} \mathrm{~mol} \mathrm{~L}^{-1}$, bromure de dodécyltriméthylammonium $5 \times$ $10^{-4} \mathrm{~mol} \mathrm{~L}^{-1}, \mathrm{pH} 4,2$. Concentration de la solution injectée $(100 \mu \mathrm{g}$ $\mathrm{ml} \mathrm{L}^{-1}$ ). Capillaire en silice fondue: longueur : $45 \mathrm{~cm}$; diamètre intérieur: $75 \mu \mathrm{m}$. Détection à $250 \mathrm{~nm}$. Tension appliquée : $20 \mathrm{kV}$. Température : $25^{\circ} \mathrm{C}$. métrie. Une séparation type est représentée figure 12 : le triphosphate, le plus mobile, sort en tête suivi du diphosphate et de l'orthophosphate. En dépit d'une ligne de base complexe les courbes d'étalonnage sont linéaires dans les domaines de concentrations $10-300 \mu \mathrm{g} \mathrm{mL} \mathrm{m}^{-1}$ pour l'orthophosphate, $50-300 \mu \mathrm{g} \mathrm{mL} \mathrm{m}^{-1}$ pour le diphosphate, $100-300 \mu \mathrm{g} \mathrm{mL} \mathrm{m}^{-1}$ pour le triphosphate avec une répétabilité de 2 à $3 \%$.

La méthode a été améliorée quant à la qualité de la ligne de base et la limite de détection en utilisant comme électrolyte une solution d'adénosine 5'-triphosphate [33] ou de ribonucléotide [34] qui absorbent également dans l'ultraviolet et permettent une détection par diminution d'absorbance. L'électrolyte contient du bromure de cétyltriméthylammonium pour inverser le flux électroosmotique ; son $\mathrm{pH}$ est de 3,5. L'injection est effectuée en mode hydrostatique du côté de la cathode, la détection étant effectuée du côté anodique. Un exemple de séparation des anions orthophosphate, diphosphate et triphosphate est représenté figure 13 avec celle d'anions polyaminocarboxylates et de citrate (de tels mélanges sont mis en œuvre dans les détergents). La ligne de base est parfaite. Les limites de détection pour les phosphates sont : $2,5 \times 10^{-5} \mathrm{~mol} \mathrm{~L}^{-1}$ pour l'orthophosphate, $2 \times$ $10^{-5}$ mol L-1 pour le diphosphate, $5 \times 10^{-5} \mathrm{~mol} \mathrm{~L}^{-1}$ pour le triphosphate.

L'électrophorèse capillaire apparaît comme étant une méthode rapide et sensible pour les premiers termes des polyphosphates linéaires. Elle ne semble pas pouvoir s'appliquer aux termes supérieurs des polyphosphates où la chromatographie d'échange d'ions reste inégalée.

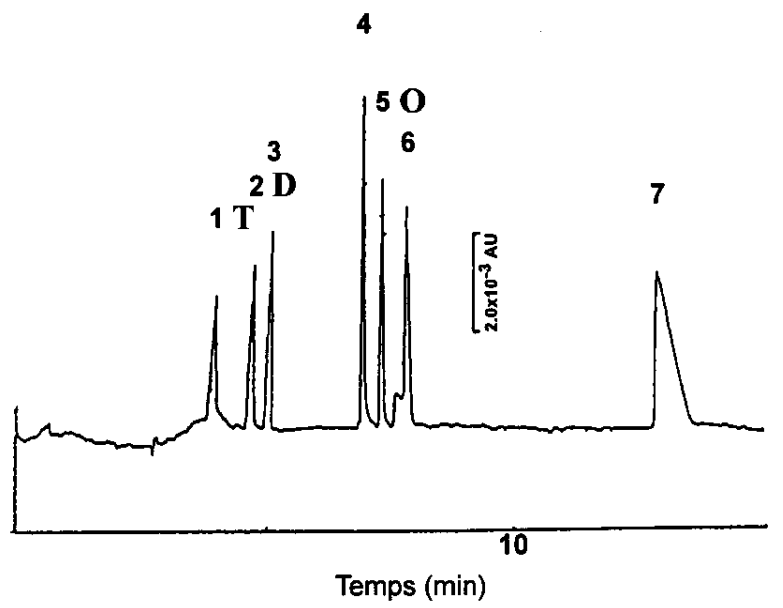

Fig. 13. Séparation des premiers termes des phosphates $(O, D, T)$ et d'acides polyaminocarboxyliques et citrique par électrophorèse capillaire. Electrolyte : adénosine 5'-triphosphate $10^{-2} \mathrm{~mol} \mathrm{~L}^{-1}$, bromure de cétyltriméthylammonium $5 \times 10^{-5} \mathrm{~mol} \mathrm{~L}^{-1}, \mathrm{pH} 3,5$. Identification des pics : $1:$ triphosphate $(\mathrm{T}) ; 2$ : diphosphate (D); $3:$ nitrilotriacétate ; $4:$ éthylènediaminetétracétate $; 5:$ orthophosphate $(\mathrm{O}) ; 6$ : diéthylènetriaminepentaacétate ; 7 : citrate. Concentration de la solution injectée : $5 \times 10^{-4} \mathrm{~mol} \mathrm{~L}^{-1}$ sauf pour le citrate : $10^{-3} \mathrm{~mol} \mathrm{~L}^{-1}$. Capillaire : longueur : $50 \mathrm{~cm}$; longueur effective : $40 \mathrm{~cm}$; diamètre intérieur : $75 \mu \mathrm{m}$. Détection à $260 \mathrm{~nm}$. Tension appliquée : $15 \mathrm{kV}$. Température: $25 \pm 1^{\circ} \mathrm{C}$ 


\section{Review}

\section{Conclusion}

La chromatographie d'échange d'ions avec élution graduée et détection absorptiométrique permet d'analyser de manière remarquable les mélanges complexes de polyphosphates linéaires (jusqu'à $\mathrm{P}_{36}$ environ) et cycliques (jusqu'à $\mathrm{P}_{8 m}$ ). Mais, les séparations durent plusieurs heures et le système de détection exige un appareillage spécialisé pour la formation du bleu de molybdène.

La chromatographie ionique a apporté un progrès considérable en abaissant à moins de 30 minutes la durée de la séparation et en utilisant une détection conductométrique pour laquelle des appareils commerciaux récents existent. Toutefois, l'analyse quantitative précise des différents phosphates pose problème en raison de l'incertitude où l'on est des coefficients de réponse des conductomètres pour les différents phosphates condensés.

L'électrophorèse capillaire conduit à des analyses d'une grande rapidité (moins de 10 minutes) mais elle est limitée, pour le moment, aux premiers termes des phosphates : ortho, di et triphosphate.

\section{Références}

1. Ohashi, S. Pure Appl. Chem. 1975, 44, 415-438.

2. Grzmil, B.; Glablisz, U. Polish J. Appl. Chem. 1991, 35, 6571.

3. Averbuch-Pouchot, M. T.; Durif, A. Topics in phosphate chemistry, World Scientific, Singapore, 1996.

4. Beukenkamp, J.; Rieman, W. III; Lindenbaum, S. Anal. Chem. 1954, 26, 505-512.

5. Grande, J. A.; Beukenkamp, J. Anal. Chem. 1956, 28, 14971498.

6. Lundgren, D. P.; Loeb, N. P. Anal. Chem. 1961, 33, 366-370.

7. Benz, C.; Paixao, L. M. Chim. Anal. 1968, 50, 247-250.

8. Benz, C. Analusis 1973, 2, 56-58.

9. Fukuda, T.; Nakamura, T.; Ohashi, S. J. Chromatogr. 1976, 128, 212-217.

10. Nakamura, T.; Yano, T.; Fujta, A.; Ohashi, S. J. Chromatogr. 1977, 130, 384-386.

11. Nakamura, T.; Yano, T.; Nunokawa, T.; Ohashi, S. J. Chromatogr. 1978, 161, 421-423.
12. Yamagushi, H.; Nakamura, T.; Hirai, Y.; Ohashi, S. J. Chromatogr. 1979, 172, 131-140.

13. Hirai, Y.; Yoza, N.; Ohashi, S. J. Chromatogr. 1980, 115, 269277.

14. Yoza, N.; Ito, K.; Hirai, Y.; Ohashi, S. J. Chromatogr. 1980, 196, 471-480.

15. Brazell, R. S.; Holmberg, R. W.; Moneyhun, J. H. J. Chromatogr. 1984, 290, 163-172.

16. Biggs, W. R.; Gano, J. T.; Brown, R. J. Anal. Chem. 1984, 56, 2653-2657.

17. Yoza, N.; Hirano, H.; Baba, Y.; Ohashi, S. J. Chromatogr. 1985, 325, 385-393.

18. Baba, Y.; Yoza, N.; Ohashi, S. J. Chromatogr. 1985, 348, 2737.

19. Baba, Y.; Yoza, N.; Ohashi, S. J. Chromatogr. 1985, 350, 119125.

20. Baba, Y.; Yoza, N.; Ohashi, S. J. Chromatogr. 1985, 350, 461467.

21. Baba, Y.; Kura, G. J. Chromatogr. 1991, 550, 5-14.

22. Linares, P.; Luque de Castro, M. D.; Valcárcel, M. J. Chromatogr. 1991, 585, 267-271.

23. Kura, G.; Kitamura, E.; Baba, Y. J. Chromatogr. 1993, 628, 241-246.

24. Shimizu, N.; Kumagai, H. Industrial Water 1993, 421, 52-56 (en japonais).

25. Wong, D.; Jandik, P.; Jones, W. R.; Hagenaars, A. J. Chromatogr. 1987, 389, 279-285.

26. Shamsi, S. A.; Danielson Neil, D. J. Chromatogr. A 1993, 653, 153-160.

27. Stover, F. S.; Bulmahn, J. A.; Gard, J. K. J. Chromatogr. A 1994, 688, 89-95.

28. Jandera, P.; Churácek, J. Chromatogr. 1974, 91, 223-226.

29. Wang, D.; Zhou, Y.; Xue, D.; Zhao, G.; Huang, H. Fenxi Ceshi Xuebao 1995, 14, 97-99 (en chinois).

30. Kouchiyama, K.; Yoza, N.; Ohashi, S. J. Chromatogr. 1978, 147, 271-279.

31. Rosset, R.; Caude, M.; Jardy, A. Chromatographies en phases liquide et supercritique, Masson, Paris, 1991, chapitre XI.

32. Stover, F. S.; Keffer, S. S. J. Chromatogr. A 1993, 657, 450454.

33. Wang, T.; Li, S. F. Y. J. Chromatogr. A 1996, 723, 197-205.

34. Shamsi, S. A.; Danielson, N. D. Anal. Chem. 1995, 67, 18451852. 